\title{
COUPLING OF SURFACE AND BULK VIBRATIONS IN THE NUCLEAR BREATHING MODE
}

\author{
M. BRACK \\ Institut für Theoretische Physik, Universität Regensburg, D-8400 Regensburg, W. Germany \\ and \\ W. STOCKER
}

Sektion Physik der Universität München, D-8046 Garching, W. Germany

Received 11 April 1983

\begin{abstract}
We treat a general class of dynamical couplings of the surface to the bulk of a spherical nucleus during isoscalar breathing vibrations. Compressibilities are evaluated in the extended Thomas-Fermi approximation using a realistic Skyrme force $\left(\mathrm{SkM}^{*}\right)$. Inertial parameters are obtained in a hydrodynamical framework. The lower of the two eigenmodes is found to be close in energy to the pure bulk vibration (and to the scaling mode), and to be in excellent agreement with the experimental giant monopole resonance energies. The coupling of surface and bulk vibrations shifts the pure surface mode to appreciably higher energies $\left(\sim 25 \mathrm{MeV}\right.$ in $\left.{ }^{208} \mathrm{~Pb}\right)$. This upper mode which has a pronounced anti-scaling behaviour, might be identified through the analysis of its transition density.
\end{abstract}

\section{Introduction}

Only in the last 2-3 years has the nuclear breathing mode been firmly established experimentally and observed systematically for some 25-30 nuclei over the whole mass table. [For a review of the experimental situation of nuclear giant resonances, see ref. ${ }^{1}$ ).] The peak energies of the (isoscalar, $T=0$ ) giant monopole resonance (GMR) follow approximately the law

$$
E_{\mathrm{GMR}} \approx 80 \mathrm{MeV} \times A^{-1 / 3} \text {. }
$$

This experimental result has been theoretically explained using different quantum-mechanical tools such as RPA calculations ${ }^{1-5}$ ), the generator coordinate method ${ }^{6}$ ) or the sum rule approach ${ }^{7}$ ). [For a review of the different theories and their close connections, see ref. ${ }^{2}$ ).] On the other hand, classical hydrodynamical or fluid dynamical models ${ }^{8-11}$ ) are also able to reproduce quantitatively the GMR energies eq. (1). A common essential ingredient in all these theoretical descriptions is the use of an effective nucleon-nucleon inter action (e.g. of Skyrme type) which must necessarily lead to an incompressibility of infinite nuclear matter $K_{\infty}$ of $\sim 200-250 \mathrm{MeV}$. 
What one actually measures in the nuclear breathing mode is not $K_{\infty}$, but the incompressibility $K_{A}$ of the finite nucleus under consideration. The quantity $K_{A}$ may be extracted from the experiment eq. (1) by the frequently used definition

$$
E_{\text {GMR }}=\sqrt{\frac{\hbar^{2}}{m} \frac{K_{A}}{\left\langle r^{2}\right\rangle}},
$$

where $\left\langle r^{2}\right\rangle$ is the mean squared radius of the nucleus. On the other hand, theoretically $K_{A}$ is a model-dependent quantity. Two models have in particular been discussed $^{3,4,11}$ ) for the breathing mode: the so-called scaling model and the constrained Hartree-Fock (HF) model. In the former, the time dependent single-particle wave functions (and, consequently the densities) are obtained from the static ones by a scale transformation

$$
\boldsymbol{r} \rightarrow \lambda \boldsymbol{r},
$$

where $\lambda$ is supposed to be a periodic time-dependent collective parameter. The ("scaled") incompressibility $K_{A}^{\mathrm{s}}$ is then obtained from the total intrinsic (e.g. HF) energy $E_{\text {ir.t }}$ by

$$
K_{A}^{\mathrm{S}}=\left.\frac{\mathrm{d}^{2}\left(E_{\text {intr }}(\lambda) / A\right)}{\mathrm{d} \lambda^{2}}\right|_{\lambda=1} .
$$

In the latter model, the nucleus is constrained (by a time-dependent external field) to have a given mean squared radius $\left\langle r^{2}\right\rangle$, and the ("constrained") incompressibility $K_{A}^{\mathrm{C}}$ is found by

$$
K_{A}^{\mathrm{C}}=\left.4 \frac{\left\langle r^{2}\right\rangle^{2}}{\mathrm{~d}\left\langle r^{2}\right\rangle / \mathrm{d} \varepsilon}\right|_{\varepsilon=0}
$$

where $\varepsilon$ is the Lagrange multiplier used to constrain $\left\langle r^{2}\right\rangle$.

The two incompressibilities $K_{A}^{\mathrm{S}}$ (eq. (4)) and $\boldsymbol{K}_{A}^{\mathrm{C}}$ (eq. (5)) are different, although closely related to each other by sum rules ${ }^{4,11}$ ). In particular, Jennings and Jackson ${ }^{4}$ ) pointed out that they have different limits for $A \rightarrow \infty$ (see eq. (7) below). However, numerical calculations for finite nuclei by Treiner et al. ${ }^{11}$ ) show that they differ only little for $40 \leqslant A \leqslant 300, K_{A}^{\mathrm{C}}$ being some $3-8 \%$ smaller than $K_{A}^{\mathrm{S}}$, so that peak energy considerations alone do not allow to decide between the two models and a more detailed analysis of transition densities is necessary.

A convenient parametrization of $K_{\mathrm{A}}$ may be introduced ${ }^{3,4}$ ) by its liquid-drop-type expansion:

$$
K_{\mathrm{A}}=K_{\mathrm{V}}+K_{\mathrm{S}} A^{-1 / 3}+K_{\delta}\left(\frac{N-Z}{A}\right)^{2}+K_{\mathrm{Coul}} Z^{2} A^{-4 / 3}+\cdots
$$

Here onc finds ${ }^{4}$ )

$$
K_{\mathrm{V}}^{\mathrm{S}}=K_{\infty}=\frac{10}{7} K_{\mathrm{V}}^{\mathrm{C}}
$$


In the scaling model, the expansion eq. (6) converges well, whereas for the constrained model curvature $\left(\sim A^{-2 / 3}\right)$ and even higher-order terms are necessary to reproduce the results of medium and lighter nuclei. We refer to the abovementioned literature ${ }^{3,4,11}$ ) for detailed discussions of the expansion eq. (6) in both models.

The object of our present investigation is a generalisation of the scaling model, allowing us to study the dynamical coupling of surface and bulk vibrations of the breathing nucleus. We shall use a semiclassical model in which the nuclear incompressibilities corresponding to the different breathing modes are obtained from variational extended Thomas-Fermi (ETF) calculations ${ }^{12-14}$ ), and the corresponding inertial parameters are obtained by solving the hydrodynamical continuity equation. We shall use a Skyrme interaction, hereafter labelled $\mathrm{SkM}^{*}$, which was recently adjusted to yield excellent fits of ground-state binding energies, radii and densities of stable spherical nuclei through HF calculations ${ }^{15}$ ), and to yield at the same time the correct average fission barriers of actinide nuclei through variational ETF calculations ${ }^{14,15}$ ). [Actually, the force $\mathrm{SkM}^{*}$ is an only slightly modified version of the one labelled SkM, which was adjusted to $0^{+}, 1^{-}$and $2^{+}$giant resonance energies in RPA-type calculations ${ }^{16}$ ).]

In preceding papers, we analyzed the static compressibility of nuclei in the variational ETF approach ${ }^{17}$ ) and collaborated in a similar study using the constrained HF approach ${ }^{18}$ ). In particular, we presented in ref. ${ }^{17}$ ) an analytically soluble "pocket model" for symmetric, semi-infinite nuclear matter, in which a whole class of compression modes can be treated where the coupling between surface and bulk compression is described by a continuous parameter $\beta$. We found that, independently of the details of the effective force used, the static surface incompressibility is minimal for an "anti-scaling" mode in which the surface becomes more diffuse when the bulk is compressed, in contrast to the scaling model mentioned above. We already anticipated in ref. ${ }^{17}$ ) that inertial effects in a dynamical treatment can shift this statically favoured anti-scaling mode towards a more scaling-like behaviour such as it seems to be supported by RPA calculations ${ }^{3}$ ).

In sect. 2 we briefly recall the basic idea of the "pocket model" of ref. ${ }^{17}$ ) and show how the static nuclear compressibilities obtained there analytically are modified by the inclusion of asymmetry and Coulomb effects. In sect. 3 we present the calculation of inertial parameters corresponding to the various modes, solving the hydrodynamical continuity equation, and discuss the corresponding velocity fields. In sect. 4 we present the resulting breathing mode frequencies. We show in particular how the choice of the surface-to-bulk coupling parameter $\beta$ is connected to the diagonalization in a two-dimensional Hilbert space, i.e. to finding the eigenfrequencies of two coupled harmonic oscillators. The lower of the two frequencies obtained is shown to be in excellent agreement with the experimental GMR peak energies throughout the mass table. 


\section{Calculation of static nuclear compressibilities}

We describe the density profile of the compressed spherical nucleus by a Fermi function

$$
\rho(r, q)=\frac{\rho_{\mathrm{c}}(q)}{\left[1+\mathrm{e}^{(r-\bar{R}) / \alpha_{\mathrm{c}}(q)}\right]} .
$$

The parameter $q$ is a measure for the compression of the nucleus and is defined as the ratio of the compressed central density $\rho_{\mathrm{c}}$ to its equilibrium value $\rho_{0}$ at $q=1$ :

$$
\rho_{\mathrm{c}}(q)=\rho_{0} q
$$

The surface diffuseness $\alpha_{\mathrm{c}}$ is related to its equilibrium value $\alpha_{0}$ by

$$
\alpha_{c}(q)=\alpha_{0} q^{\beta}
$$

here $\beta$ is a real, dimensionless parameter which allows us to control the degree of coupling between surface and bulk compression, thus defining a whole class of different compression modes. For $\beta=0$ we have a pure bulk compression with $\alpha_{c}=\alpha_{0}$ remaining unchanged; $\beta=-\frac{1}{3}$ corresponds to the scaling mode, and in the limit $\beta \rightarrow \pm \infty$ we have a pure surface mode. In ref. ${ }^{17}$ ) we have shown that in the limit $R \rightarrow \infty$, where the one-dimensional geometry of semi-infinite nuclear matter applies, the profile defined by eqs. (8)-(10) is found as an analytical solution to the variational Euler equation with a simplified, but realistic model energy density (incorporating the correct saturation properties $\rho_{\infty},(E / A)_{\infty}, K_{\infty}$ as well as the surface energy coefficient $a_{\mathrm{s}}$ ) and an external constraint which depends parametrically on $\beta$.

For finite nuclei, the radius $R$ must be adjusted to give the correct nucleon number and thus is a function of $\rho_{0}, \alpha_{0}, q$ and $\beta$. In a leptodermous expansion (see appendix A) it is found to be

$$
R=r_{0}(q) A^{1 / 3}-\left(\frac{1}{3} \pi^{2} \alpha_{0}^{2} q^{2 \beta} / r_{0}(q)\right) A^{-1 / 3}+\cdots,
$$

with

$$
r_{0}(q)=\left(\frac{4}{3} \pi \rho_{0}\right)^{-1 / 3} q^{-1 / 3}
$$

Truncation of the expansion (11) after the second term is sufficient to give the nucleon numbers within $\leqslant 10^{-4}$ for $A \geqslant 40$ with realistic values of $\alpha_{0}$ and $\rho_{0}$. Since we are interested here only in isoscalar modes, we do not differentiate between neutrons and protons in our formulae. However, in all numerical calculations presented below we used different equilibrium values $\alpha_{0}$ and $\rho_{0}$ for neutrons and protons. [Their values may be found in refs. $\left.{ }^{14,15}\right)$.]

Our picture of the vibrating nucleus is thus the following. We treat the compression ratio $q$ of the bulk density as a (periodically time-dependent) collective parameter, and fix the surface-to-bulk coupling parameter $\beta$ in a kind of 
"freezing approximation". As we shall discuss explicitly in sect. 4, no dynamical information is hereby lost about the system, possessing basically two independent degrees of freedom (namely, $\rho_{\mathrm{c}}$ and $\alpha_{\mathrm{c}}$ ), if $\beta$ is determined at the end by a variational principle. Thus, we could as well have started by treating $\alpha_{\mathrm{c}}$ and $\rho_{\mathrm{c}}$ as independent dynamical variables. However, we prefer at the present stage to stay with the above picture with $\beta$ as a frozen parameter, since it allows for a very transparent interpretation of the coupling of surface and bulk vibrations and, in particular, of the connection with the particular mode described in the scaling model $\left(\beta=-\frac{1}{3}\right)$.

Restricting ourselves to small amplitude vibrations, we have to treat the dynamics of a harmonic oscillator. Its frequency is given by a restoring force parameter $K$ and an intertial parameter $B$ (to be discussed in the next section), both of which depend on $\beta$ :

$$
\omega^{2}=K(\beta) / B(\beta) .
$$

The quantity $K(\beta)$ is just the static compressibility of the nucleus, defined by

$$
K(\beta)=\left.9 \frac{\mathrm{d}^{2}}{\mathrm{~d} q^{2}}\left(E_{\text {intr }} / A\right)\right|_{\substack{\beta \text { fixed } \\ q=1}},
$$

where $E_{\text {intr }}$ is the total intrinsic energy of the nucleus, which here is calculated in a semiclassical way. The definition of $K(\beta)$ (eq. (14)) is identical with that of $K_{A}^{\mathrm{S}}$ (eq. (4)) for the scaling mode (with $\beta=-\frac{1}{3}$ and $\lambda=q^{1 / 3}$ ); it leads to the infinite nuclear matter incompressibility $K_{\infty}$ in the limit $A \rightarrow \infty$ (i.e. $R \rightarrow \infty$ ):

$$
K(\beta) \underset{A \rightarrow \infty}{\longrightarrow} K_{\infty}
$$

for all values of $\beta$.

As mentioned in the introduction, we calculate the total intrinsic energy of the compressed nucleus in a variational energy-density approach, using the extended Thomas-Fermi (ETF) model ${ }^{12-14}$ ) and the Skyrme force SkM*14,15$)$. The energy is thus written as

$$
E_{\text {intr }}=\int \mathrm{d}^{3} r \mathscr{E}_{\mathrm{Sky}}\left[\rho_{\mathrm{n}}, \rho_{\mathrm{p}}\right]+E_{\mathrm{Coul}}\left[\rho_{\mathrm{p}}\right] .
$$

Here, the complete (and parameter free!) ETF functionals $\tau[\rho]$ and $\boldsymbol{J}[\rho]$ up to fourth order in $\hbar\left[\right.$ refs $\left.{ }^{19.20}\right)$ ] for the kinetic energy and spin-orbit densities, respectively, are used, including the corrections due to the spin-orbit force and the variable effective nuclear masses $m^{*}(r)$. [For details of the Skyrme functional $\mathscr{E}_{\mathrm{Sky}}\left[\rho_{\mathrm{n}}, \rho_{\mathrm{p}}\right.$ ] obtained in this way, see ref. ${ }^{14}$ ).] The second item in eq. (16) is the Coulomb energy, whose direct part is integrated exactly and the exchange part is calculated in the usual local density (Slater) approximation. The densities $\rho_{\mathrm{n}}, \rho_{\mathrm{p}}$ are parametrized according to eq. (8) (with different values for $\rho_{0}, \alpha_{0}$ and $R$ for neutrons and protons), and the total energy (eq. (16)) is minimized with respect to the 
variational density parameters. This method has recently been shown to lead to an excellent description of average nuclear ground state properties (binding energies, densities and radii) and deformation energies, in close agreement with selfconsistently averaged Hartree-Fock results ${ }^{14,15}$ ).

We calculated the incompressibility $K(\beta)$ (eq. (14)) numerically by performing small isoscalar compressions of the densities, according to eqs. (9), (10), around their equilibrium shapes. It is not necessary to repeat this for each given value of $\beta$; in fact it is easily seen that $K(\boldsymbol{\beta})$ is of a pure parabolic form. If we introduce infinitesimally small independent bulk and surface-diffuseness compressions by defining

$$
q_{\rho}=\frac{\rho_{\mathrm{c}}}{\rho_{0}}=1+\delta q_{\rho}, \quad q_{\alpha}=\frac{\alpha_{\mathrm{c}}}{\alpha_{0}}=1+\delta q_{\alpha},
$$

it follows from the definition (eq. (10)) for $\beta$ that

$$
\delta q_{\alpha}=\beta \delta q_{\rho} .
$$

Expanding the energy around its equilibrium value $E_{0}$,

$$
E_{\mathrm{intr}}=E_{0}+\frac{1}{9} A\left\{\frac{1}{2} K_{\rho \rho}\left(\delta q_{\rho}\right)^{2}+K_{\alpha \rho} \delta q_{\rho} \delta q_{\alpha}+\frac{1}{2} K_{\alpha \alpha}\left(\delta q_{\alpha}\right)^{2}\right\},
$$

it follows that we can write $K(\beta)$ in the form

$$
K(\beta)=K_{\rho \rho}+2 \beta K_{\alpha \rho}+\beta^{2} K_{\alpha \alpha} .
$$

Thus, all we have to know for a given nucleus is the symmetric matrix of compression moduli $K_{i j}(i, j=\alpha, \rho)$. The "scaled" incompressibility $K_{A}^{\mathrm{S}}$ (eq. (4)) e.g. is given by

$$
K_{A}^{\mathrm{S}}=K_{\rho \rho}-\frac{2}{3} K_{\alpha \rho}+\frac{1}{9} K_{\alpha \alpha} .
$$

It is illustrative to discuss $K(\beta)$ and its constituents $K_{i j}$ in terms of their liquiddrop-type expansion (eq. (6)). Keeping only the volume and isoscalar surface terms, one obtains

$$
K(\beta)=K_{\infty}+4 \pi r_{0}^{2}\left\{22 \sigma_{0}+9 \ddot{\sigma}_{0}(\beta)+54\left(\sigma_{0} / K_{\infty}\right) \dddot{e}_{0}\right\} A^{-1 / 3},
$$

where $\sigma_{0}$ is the surface tension at the saturation density, $\ddot{\sigma}_{0}$ is its second derivative with respect to $q$,

$$
\ddot{\sigma}_{0}(\beta)=\left.\frac{\mathrm{d}^{2}}{\mathrm{~d} q^{2}} \sigma\left(\rho_{\mathrm{c}}, \alpha_{\mathrm{c}}\right)\right|_{\substack{\beta \text { fixed } \\ q=1}},
$$

and $\dddot{e}_{0}$ is the anharmonicity coefficient of the equation of state at the saturation point $\left(\ddot{e}_{u}--14.4 \mathrm{MeV}\right.$ for the $\mathrm{SkM}^{*}$ force). Eq. (21) was originally derived for the scaling mode ${ }^{3}$ ), but it holds for arbitrary values of $\beta$. In the schematic pocket model of ref. ${ }^{17}$ ), where asymmetry, Coulomb and curvature corrections were 
omitted, we derived the following expression for $\ddot{\sigma}_{0}(\beta)$ :

$$
\ddot{\sigma}_{v}(\beta)=\sigma_{v}\left(\beta^{2}-2 \beta-5\right),
$$

which turned out to be approximately fulfilled also for various realistic Skyrme forces and energy densities with different values of $K_{\infty}$ and $\sigma_{0}$. From eqs. (21) and (23), we can immediately read off the volume and surface terms of the compression moduli $K_{i j}$ :

$$
\begin{aligned}
K_{p \rho} & \approx K_{\infty}+a_{\mathrm{s}}\left(-23+54 \ddot{e}_{0} / K_{\infty}\right) A^{-1 / 3}, \\
K_{\alpha \rho} & \approx-9 a_{\mathrm{s}} A^{-1 / 3}, \quad K_{\alpha \alpha} \approx 9 a_{\mathrm{s}} A^{-1 / 3},
\end{aligned}
$$

where $a_{\mathrm{s}}=4 \pi r_{0}^{2} \sigma_{0}$ is the usual surface energy coefficient $\left[a_{\mathrm{s}}=17.5 \mathrm{MeV}\right.$ for the $\mathrm{SkM}^{*}$ force $\left.{ }^{14}\right)$ ]. It is obvious that $K_{\alpha \rho}$ and $K_{\alpha \alpha}$ have no volume term and thus vanish in the limit $\mathrm{A} \rightarrow \infty$.

In fig. 1 we present the compression moduli $K_{i j}$ obtained in the realistic ETF calculations, thus including asymmetry, Coulomb and curvature (and higher-order) effects, alongside their pocket model values, eq. (24), as functions of $A^{-1 / 3}$. The surface incompressibilities $K_{\alpha \alpha}$ and $K_{\alpha \rho}$ are seen to approach well their asymptotic behaviour from eq. (24) for $A \geqslant 200$. For lighter nuclei, the curvature and higherorder terms evidently become important. This is much less the case for the bulk

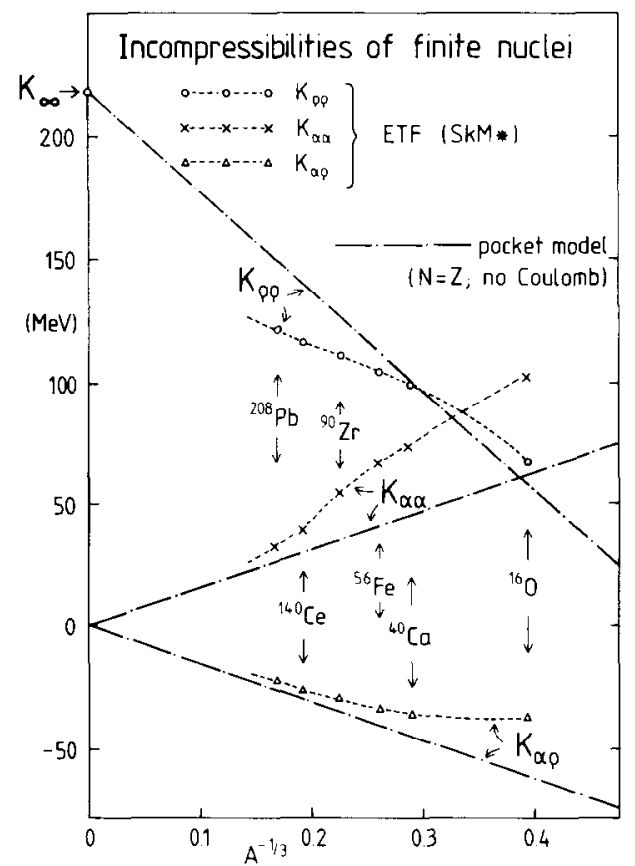

Fig. 1. Incompressibilities of finite nuclei, calculated in the variational ETF approach with the Skyrme $\mathrm{SkM}^{*}$ force, versus $A^{-1 / 3}$. The definitions of the $K_{i j}$ and their pocket model values are given in the text. 
incompressibility $K_{\rho \rho}$. That quantity is, in turn, appreciably modified for heavier nuclei by asymmetry and Coulomb effects, which both contribute negatively and have been extensively discussed in the literature ${ }^{3,4,11}$ ). For the "scaled" incompressibility $K_{A}^{\mathrm{S}}$, the curvature term (proportional to $A^{-2 / 3}$ ) has been found to be numerically negligible ${ }^{3,11,13}$ ). As we can see from the results in fig. 1 , this is rather a coincidence due to a cancellation of the curvature contributions in the particular combination of eq. (20).

In ref. ${ }^{17}$ ) we observed that the incompressibility $K(\beta)$ obtained with eqs. (21), (23) is minimal for $\beta=1$ and concluded that from a purely static point of view (thus neglecting inertial effects), an "anti-scaling" type of vibration would be energetically favoured, in which the surface is becoming more diffuse as the central density increases and vice versa, contrary to the scaling mode. This result is somewhat changed when using the realistic incompressibilities. In fact, the value $\beta_{0}$ minimizing $K(\beta)$ is from eq. (19) seen to be

$$
\beta_{0}=-K_{\alpha \rho} / K_{\alpha \alpha} .
$$

and takes values $\beta_{0} \approx 0.4-0.6$ for real nuclei, compared to the pocket model value $\beta_{0}=1$. But still, this static consideration leads to an anti-scaling of the density. It will therefore be interesting to see how the inertial effects, as anticipated in ref. ${ }^{17}$ ), drastically change this result.

Before turning to the dynamics in the next section, a word about the adequateness of our semiclassical description of the incompressibilities $K_{i j}$ might be appropriate. Because of the complete equivalence of the variational ETF model with a selfconsistently Strutinsky-averaged HF approach ${ }^{15,21}$ ), the question can be reduced to whether the quantal single particle effects (i.e. the shell effects ) play an important role for the compression moduli $K_{i j}$. This question can be answered for the scaling mode, for which the exact incompressibility $K_{A}^{\mathrm{S}}$ can be expressed analytically, in terms of the different contributions to the ground-state HF energy [see e.g. ref $^{3}$ )]. Using the standard spherical HF code of the Orsay group ${ }^{22}$ ), we calculated $K_{A}^{\text {s }}$ with the $\mathrm{SkM}^{*}$ force for the ${ }^{208} \mathrm{~Pb}$ nucleus. The (exact $\mathrm{HF}$ ) result was $K_{\mathrm{A}}^{\mathrm{S}}=$ $141 \mathrm{MeV}$, whereas the semiclassical result, obtained as described above, is $K_{A}^{\mathrm{S}}=$ $139 \mathrm{MeV}$ (see table 1 below). Since the relative accuracy of our numerically calculated $K_{i j}$ is of the order of a few percent, the agreement is thus perfect. This demonstrates that the sheil effects in $K_{A}^{\mathrm{S}}$ are below the $\sim 1 \%$ level and can therefore be safely neglected in our approach. There is no reason to suppose that this conclusion must be altered for modes with different values of $\beta$.

\section{Calculation of inertial parameters}

We now address ourselves to the question of how the inertial parameters $B(\beta)$ can be calculated and how strongly they depend on $\beta$. For the particular case of the scaling model $\left(\beta=-\frac{1}{3}\right)$, it has been proved analytically $\left.{ }^{23}\right)$ that the microscopical 
Inglis formula (without residual interaction) leads to the classical, irrotational hydrodynamical inertia given by

$$
B_{\text {scal }}=B\left(-\frac{1}{3}\right)=m\left\langle r^{2}\right\rangle_{0} q^{-8 / 3},
$$

where $\left\langle r^{2}\right\rangle_{0}$ is the mean squarc radius of the nuclcus at equilibrium $(q=1)$. Thus, there are no shell effects at all in the inertial parameter in this case. If this is to be true also for the other compression modes, which we will suppose here, we can use the hydrodynamical model to calculate the inertial parameters for other values of $\beta$ than $-\frac{1}{3}$, too.

Jensen and Larsen ${ }^{10}$ ) used a similar approach, which was originally derived from the time-dependent HF theory ${ }^{24}$ ). It turns out, however, after the elimination of some redundant quantities, that their approach is identical to the hydrodynamical model with irrotational flow $^{\dagger}$. For a rather detailed discussion of derivations of hydrodynamics from TDHF see also ref. ${ }^{29}$ ). With these justifications in mind, we set forth to define a velocity field $v(r, q)$ by writing down the hydrodynamical continuity equation:

$$
\frac{\partial \rho}{\partial q}+\nabla \cdot(\rho v)=0
$$

Note that $v$ is measured for convenience in units of the time-dependent collective velocity $\dot{q}=\mathrm{d} q(t) / \mathrm{d} t$. Due to the spherical symmetry of the breathing nucleus, the velocity field has only a radial component $v(r, q)$.

In a self-consistent hydrodynamical [or fluid dynamical $\left.{ }^{9}\right)$ ] approach, $\rho(r, q)$ and $v(r, q)$ would be found by simultaneously solving eq. (27) and the dynamical Euler equation. In our present model, however, we have imposed the dynamics by the explicit definition, eqs. (8)-(10), of the $q$-dependence of the density $\rho(r, q)$. Therefore, $v(r, q)$ is already determined from the continuity equation (27). This equation can, in fact, be integrated in the case of spherical symmetry to yield ${ }^{3,10}$ )

$$
v_{\beta}(r, q)=-\left.\frac{1}{r^{2} \rho(r, q)} \int_{0}^{r} r^{\prime 2} \frac{\partial \rho\left(r^{\prime}, q\right)}{\partial q}\right|_{\beta \text { fixed }} \mathrm{d} r^{\prime} .
$$

In terms of the velocity field, the inertial parameter is given by

$$
B_{q}(\beta)=\frac{9}{A} m \int \rho(r, q) v_{\beta}^{2}(r, q) \mathrm{d}^{3} r
$$

where the factor $9 / A$ was introduced for convenience analogously to eq. (14).

Starting from eq. (8) for the densities $\rho(r, q)$ with the equilibrium values of $\alpha_{0}$ and $\rho_{0}$, we have calculated $v_{\beta}(r, q)$ and $B_{q}(\beta)$ using numerical quadratures. As the incompressibility $K(\beta)$, the inertial parameter $B_{q}(\beta)$ can be written in the form

$$
B_{q}(\beta)=B_{\rho \rho}+2 \beta B_{\rho \alpha}+\beta^{2} B_{\alpha \alpha},
$$

We are grateful to Dr. J. Libert for his assistance in working out this equivalence. 
since it is quadratic in the derivative $\partial / \partial q$ with fixed $\beta$. The inertial tensor $B_{i j}$ is here given by

$$
B_{i j}=\frac{9}{A} m \int \rho v_{i} v_{j} \mathrm{~d}^{3} r \quad(i, j=\rho, \alpha),
$$

whereby $v_{\rho}(r, q)$ and $v_{\alpha}(r, q)$ are defined as in eq. (28), but holding, respectively, $\alpha_{\mathrm{c}}$ and $\rho_{\mathrm{c}}$ fixed when deriving $\rho(r, q)$ with respect to $q$. Thus, $B_{\alpha \alpha}$ is the inertia for a pure surface vibration and $B_{\rho \rho}$ that for a pure bulk density vibration. For the scaling mode $\left(\beta=-\frac{1}{3}\right)$, eq. (28) can be integrated analytically to yield

$$
v_{\text {scal }}(r, q)=-(1 / 3 q) r
$$

which, inserted into eq. (29), gives the scaling inertia $B_{\text {scal }}$ (eq. (26)).

In fig. 2 we show for ${ }^{208} \mathrm{~Pb}$ the resulting velocity fields $v(r)$ for various values of $\beta$ as well as for the pure surface mode (all at $q=1$ ). Also shown in fig. 2 is the total density $\rho(r)$ at equilibrium. It is interesting to note that for all finite values of $\beta$, the velocity fields $v(r)$ go like that of the scaling mode, eq. (32), in the nuclear interior where the density is essentially constant, whereas in the extcrior wherc $\rho(\mathrm{r})$ is exponentially small, they diverge linearly with slopes equal to $\beta$ :

$$
v(r) \sim v_{0}+\beta r \quad(r \gg R) .
$$

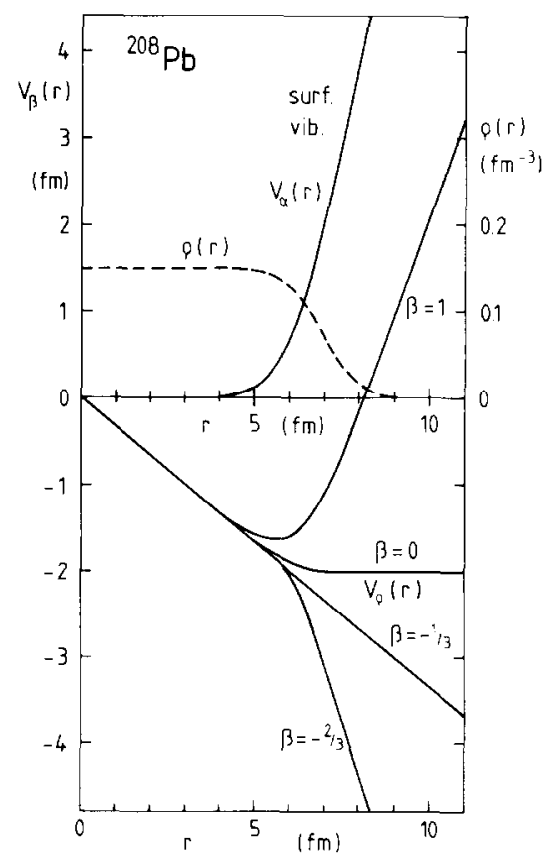

Fig. 2. Velocity fields $v_{\beta}(r)$ for different breathing modes, evaluated from the variational ETF groundstate density of ${ }^{208} \mathrm{~Pb}$ (dashed curve, scale on the right-hand side) as defined in the text. Note the linear asymptotic behaviour of $v_{\beta}(r)$ (except for $v_{\alpha}(r)$ ), which is derived also in the appendix A. 
Eq. (33) and an approximate value of $v_{0}$ are derived in appendix A. For the pure surface vibration, $v(r)$ is nonzero only in the surface region and increases exponentially there.

The fact that $B_{\rho \alpha}$ and $B_{\alpha \alpha}$ are by no means negligible with respect to $B_{\rho \rho}$ (see also table 1 below) leads to a rather strong variation of $B_{q}$ with $\beta$. The tendency is that for a fixed nucleus, $B_{\mathrm{q}}(\beta)$ has a minimum for $\beta \sim 1-2$ and increases when $\beta$ goes to smaller values. This is demonstrated in fig. 3 where we have plotted $B_{q}$ versus the nucleon number $\boldsymbol{A}$ for different values of $\beta$. This variation of the inertia has the effect that more negative values of $\beta$ are dynamically favoured compared to the purely static result (see eq. (25)), as anticipated in ref. ${ }^{17}$ ) and discussed in more detail in the next section.

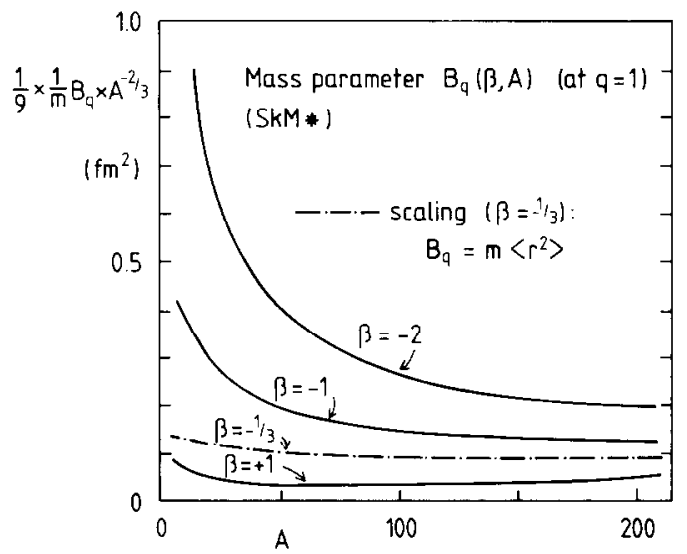

Fig. 3. Inertial parameters $B_{q}$ for various breathing modes versus nucleon number $A$.

\section{Results for the breathing frequencies}

Having calculated the incompressibility $K(\beta)$ and the inertia $B_{q}(\beta)$, we have the collective hamiltonian in the harmonic approximation:

$$
H_{q}=E_{0}+\frac{1}{2} B_{q}(\beta) \dot{q}^{2}+\frac{1}{2} K(\beta)(q-1)^{2} .
$$

After quantizing $H_{q}$ we obtain thus the spectrum of a harmonic oscillator with the frequency

$$
\hbar \omega(\beta)=\hbar \sqrt{\frac{K(\beta)}{B_{q}(\beta)}}=\sqrt{\hbar^{2} \frac{K_{\rho \rho}+2 \beta K_{\alpha \rho}+\beta^{2} K_{\alpha \alpha}}{B_{\rho \rho}+2 \beta B_{\alpha \rho}+\beta^{2} B_{\alpha \alpha}}},
$$

where both tensors $B_{i j}$ and $K_{i j}$ are evaluated at equilibrium, $q=1$.

We now have to discuss the role of the "frozen" parameter $\beta$ and, in particular, to specify its choice before identifying $\hbar \omega(\beta)$ with the energy of the breathing 
mode. Intuitively, it sounds reasonable to assume that the minimum of the function $\hbar \omega(\beta)$ corresponds to the most probable excitation energy. This is, in fact, more than a suspicion because of Rayleigh's variational principle ${ }^{25}$ ), which in the present case states the following: the two energies $\hbar \omega\left(\beta_{i}\right)=E_{i}$ of the stationary points of the function $\hbar \omega(\beta)$

$$
\left.\frac{\partial}{\partial \beta} \hbar \omega(\beta)\right|_{\beta_{i}}=0,
$$

are identical with the energies of the two normal modes of the coupled system of surface $\left(\alpha_{\mathrm{c}}\right)$ and bulk density $\left(\rho_{\mathrm{c}}\right)$ oscillations. In other words: We could as well have started from $q_{\rho}$ and $q_{\alpha}$, eq. (17a), as independent collective variables and diagonalized the corresponding hamiltonian

$$
H=\frac{1}{2} \sum_{i, j} B_{i j} \dot{q}_{i} \dot{q}_{i}+\frac{1}{2} \sum_{i, i} K_{i j}\left(q_{i}-1\right)\left(q_{j}-1\right)+E_{0} \quad(i, j=\alpha, \rho),
$$

by solving the matrix equation

$$
\left(\boldsymbol{K}-\omega^{2} B\right) \boldsymbol{x}=0 .
$$

As demonstrated explicitly in the appendix B, the eigenmodes $\omega_{i}$ of eq. (38) are then identical with those found at the stationary points of $\omega(\beta)$, eq. (36). The eigenvectors $\boldsymbol{x}_{i}$ in eq. (38) are, up to a normalization factor, given by

$$
\boldsymbol{x}_{i}=\left(\begin{array}{c}
1 \\
\beta_{i}
\end{array}\right) .
$$

Thus, we recognize the parameter $\beta$ at the stationary points eq. (36) to be equal to the ratio of the amplitude of the surface vibration to that of the bulk vibration in either of the two normal modes of the system.

This is illustrated in fig. 4 , where we have plotted $\hbar \omega(\beta)$ for the two nuclei ${ }^{208} \mathrm{~Pb}$ and ${ }^{58} \mathrm{Ni}$. The horizontal dashed lines correspond to the energies of the pure surface modes, obtained from eq. (35) in the limit $\beta \rightarrow \pm \infty$ :

$$
E_{\alpha \alpha}=\lim _{\beta \rightarrow \pm \infty} \hbar \omega(\beta)=\hbar \sqrt{K_{\alpha \alpha} / B_{\alpha \alpha}} .
$$

As we can see from the figure, $E_{\alpha \alpha}$ is shifted to appreciably higher energies, $E_{2}=\hbar \omega\left(\beta_{2}\right)$, by the coupling of the two degrees of freedom, whereas the energy $E_{1}=\hbar \omega\left(\beta_{1}\right)$ of the lower normal mode lies only a little lower than the unperturbed bulk vibration energy

$$
E_{\rho \rho}=\hbar \omega(\beta=0)=\hbar \sqrt{K_{\rho \rho} / B_{\rho \rho}} .
$$

This trend is found throughout the periodic table, as seen from table 1 in which we present the results $\beta_{i}$ and $E_{i}$ for five spherical nuclei from ${ }^{40} \mathrm{Ca}$ to ${ }^{208} \mathrm{~Pb}$. Also 


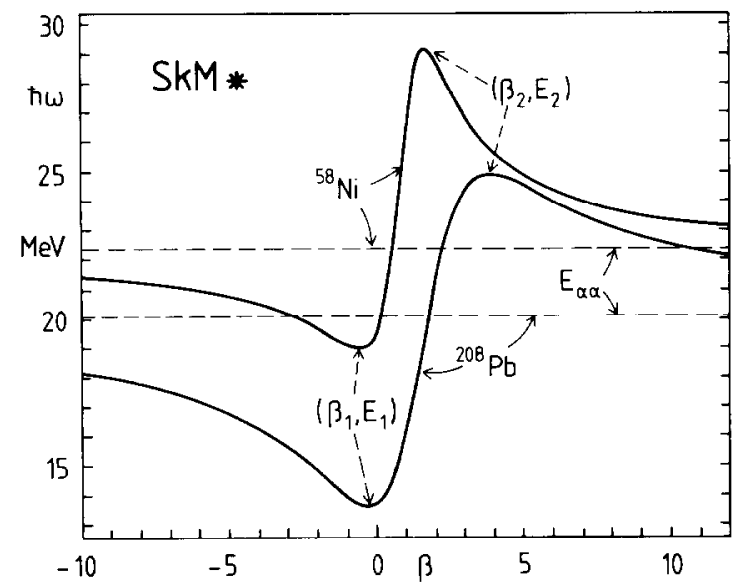

Fig. 4. Breathing mode frequencies for the two spherical nuclei ${ }^{58} \mathrm{Ni}$ and ${ }^{208} \mathrm{~Pb}$ as functions of the surface-to-bulk coupling parameter $\beta$. The horizontal dashed lines (asymptotes) are the pure surface vibrational frequencies. The stationary points give the eigenfrequencies, according to Rayleigh's variational principle as discussed in appendix $\mathbf{B}$.

shown in this table are the elements of the compression modulus $K_{i j}$ and the inertial tensor $B_{i j}$, as well as the scaling model quantities $K_{A}^{\mathrm{S}}$ and $B_{\text {scal }}=m\left\langle r^{2}\right\rangle$.

We arrive thus at the following picture. Due to the existence of (rather strongly coupled) bulk density and surface vibrations, the (isoscalar) giant monopole vibration is split into two normal modes. That the coupling is strong can be seen from the fact that the unperturbed splitting, $E_{\alpha \alpha}-E_{\rho \rho}$, is increased through the coupling by a factor $\sim 2$ in heavy to 3 in light nuclei (see fig. 4). The resulting splitting, $E_{2}-E_{1}$, is seen from table 1 to be remarkably constant, about $10-11 \mathrm{MeV}$. It is much larger than the experimental width of the GMR, $\Gamma \simeq 2-4 \mathrm{MeV}$ [ref. $\left.\left.{ }^{1}\right)\right]$, and should therefore be observable. A priori, we have within our model no knowledge of the distribution of the collective monopole strength on the two modes. A hint may be given by the fact that the higher mode is pushed up strongly by the coupling, whereas the lower mode is close in energy to the unperturbed bulk vibration. This might indicate that the upper mode carries relatively little strength, as it has been observed in similar investigations of coupled monopole and quadrupole vibrations ${ }^{23}$ ). A quantitative investigation of this point would necessitate the study of sum rules, which we will leave to a future study.

Definitely, we want to identify the lower normal mode with the experimentally known giant monopole resonance. A comparison of our results, interpolated by a smooth curve, with the experimental GMR peak energies ${ }^{1,11}$ ) is made in fig. 5. It reveals a remarkable agrecment between our calculated encrgies $E_{1}$ with the experimental peak energies within their error bars, also for light nuclei where the energies deviate significantly from the classical average trend eq. (1). 


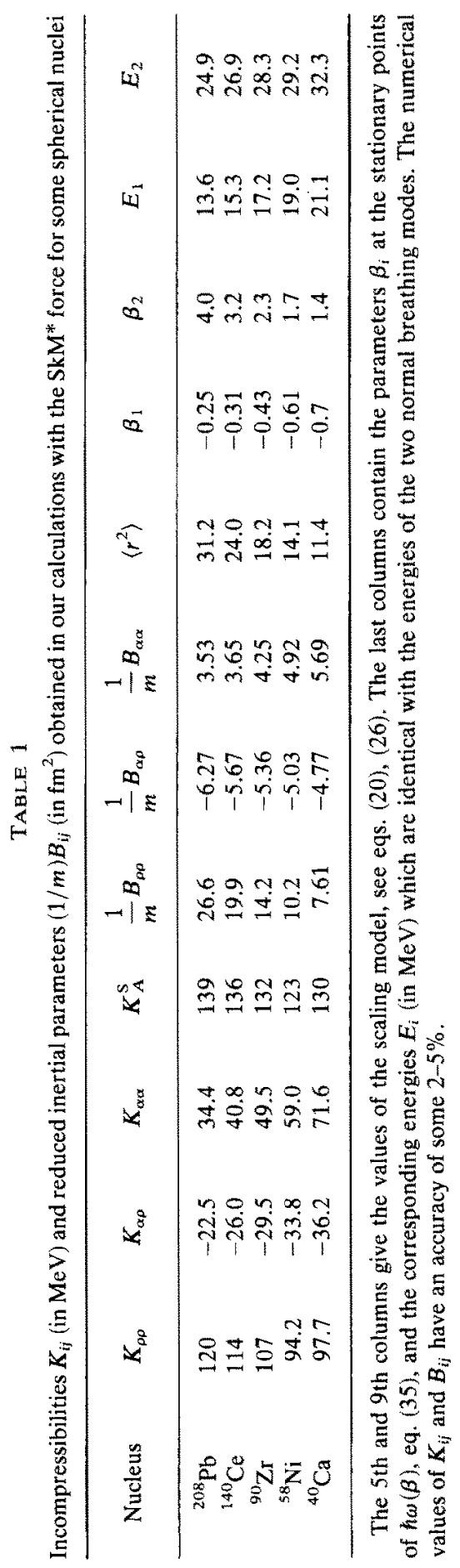




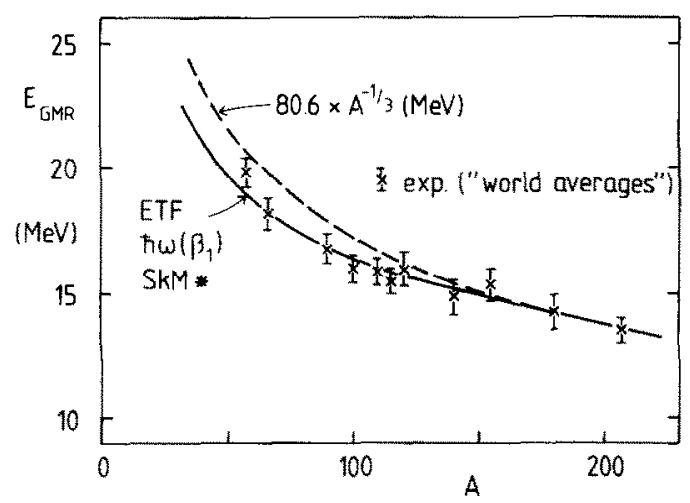

Fig. 5. Energies of the lower breathing eigenmodes of spherical nuclei, obtained in our variational semiclassical calculation using the $\mathrm{SkM}^{*}$ force (solid line). The crosses with error bars show some expcrimcntal GMR peak energies [averages of the values quoted in refs. $\left.{ }^{1,11}\right)$ ]. The dashed curve corresponds to the estimate in eq. (1).

The quality of our semiclassical results is at least competitive with what we can expect from RPA calculations if performed with the same interaction ${ }^{3,11,16}$ ). Theoretical support for the equality of the two approaches is given by the fact that the minimum of the frequency $\hbar \omega$ obtained in a variational hydrodynamical approach can be shown ${ }^{26}$ ) to be an upper bound on the energy of the lowest RPA mode.

For medium and heavy nuclei, the coupling parameter $\beta_{1}$ is found to be remarkably close to the value $-\frac{1}{3}$ of the scaling mode. This demonstrates once more the closeness of our results to those of RPA calculations which are known to be in quantitative agreement with the scaling model $^{3}$ ). For lighter nuclei, there is a clear trend of $\beta_{1}$ to go to more negative values $\left(\beta_{1}=-0.7\right.$ for $\left.{ }^{40} \mathrm{Ca}\right)$, showing that the surface has a bigger part in the breathing vibrations than predicted by the scaling model. Such a trend has been observed ${ }^{30}$ ) in the analyses of experimental cross sections in terms of transition densities. The same qualitative trend was also found in the calculations of Jensen and Larsen ${ }^{24}$ ), although these authors omitted the spin-orbit interaction and the semiclassical corrections to the Thomas-Fermi kinetic energy and could therefore not obtain the same quantitative agreement with the experiment.

The average trends of our results can also be analysed in terms of the "pocket model" (PM) described in ref. ${ }^{17}$ ) and in sect. 2 above. Since $\beta_{1}$ and $\beta_{2}$ can be expressed in terms of the components of the incompressibility tensor (see appendix B) only,

$$
\beta_{1,2}=\bar{\beta} \pm \sqrt{\bar{\beta}^{2}+1}, \quad \bar{\beta}=\frac{1}{2}\left(\beta_{1}+\beta_{2}\right)=\left(K_{\alpha \alpha}-K_{\rho \rho}\right) / 2 K_{\alpha \rho},
$$

we may use the approximate values of $K_{i j}$ eq. (24) to estimate $\bar{\beta}$ :

$$
\bar{\beta}_{\mathrm{PM}}=\frac{K_{\infty}+a_{\mathrm{s}}\left(-32+54 \dddot{e}_{\mathrm{o}} / K_{\omega}\right) A^{-1 / 3}}{18 a_{\mathrm{s}} A^{-1 / 3}},
$$




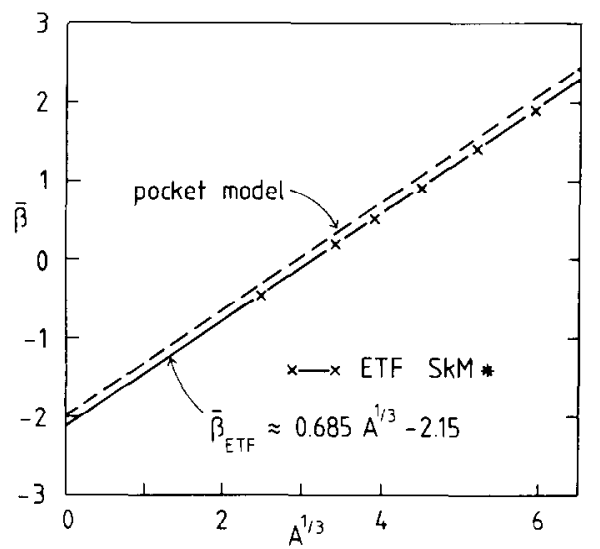

Fig. 6. Average value $\bar{\beta}$ of the surface-to-bulk amplitude ratios $\beta_{1,2}$ (eq. (39)) versus $A^{1 / 3}$. The dashed line shows the pocket model estimate eq. (43) which ignores asymmetry, Coulomb and curvature contributions. The crosses represent the realistic numerical values obtained with the $\mathrm{SkM}^{*}$ force, including all these contributions.

which, with the constants appropriate to the $\mathrm{SkM}^{*}$ force, gives

$$
\bar{\beta}_{\mathrm{PM}}=0.688 A^{1 / 3}-1.977 \text {. }
$$

In fig. 6 we haves plotted the values of $\bar{\beta}$ obtained in our ETF calculations (see table 1) versus $A^{1 / 3}$. We find, surprisingly, that these values lie on a perfect straight line which can be fitted by

$$
\bar{\beta}_{\mathrm{ETF}}=0.685 A^{1 / 3}-2.15 .
$$

Since our theoretical values of $K_{i j}$ are accurate only within a few percent, the agreement of the pocket model formula with the numerical result eq. (45) is almost quantitative; in particular, the coefficient of the leading term is exact within our numerical accuracy. This is only explainable by cancellations of Coulomb, asymmetry and curvature contributions in evaluating the mean value $\bar{\beta}$ eq. (42). The frequencies $\hbar \omega(\beta)$, however, and therefore the energies $E_{1}, E_{2}$, are sensitively affected by these contributions.

Nevertheless, eqs. (42), (43) allow a qualitative discussion of the results in asymptotic domains of the nucleon number. In the limit $A \rightarrow \infty$ we find $\beta_{1}=0$, $\beta_{2}=\infty$; the coupling of surface and bulk vibrations thus vanishes, as it is expected since it is a surface effect, and the lower mode is thus identical with the pure bulk vibration mode (and not the scaling mode!). But, as we have seen, this limit is obtained only for unphysically large values of $A$. In the other limit of very light nuclei, $\bar{\beta}$ takes values $\leqslant 0$ (for $A \leqslant 30$ ), thus indicating the trend of $\beta_{1}$ towards -1 and below, as seen in our results. For nuclei with $A<40$, however, our description 
of the density by a Fermi function is no longer well justified ${ }^{14}$ ), and we can therefore not extrapolate our results far below the $\mathrm{Ca}$ region.

\section{Summary and outlook}

One motivation for the present investigation on the dynamic coupling of the nuclear bulk and the surface came from the necessity of understanding the statically favoured antiscaling behaviour of the density of a compressed nucleus, which is in contrast to the scaled density found in RPA calculations for the dynamical breathing mode. Thus, the main question was the influence of the inertia on the structure of the dynamical density vibration. We also had to include Coulomb, asymmetry and curvature effects and a realistic nucleon-nucleon interaction.

In a hydrodynamical approach we impose a constrained $0^{+}$-density vibration on the nucleus whose density profile is taken to be of Fermi-type parametrized by two parameters: the bulk density and the surface thickness, which are connected through a parameter $\beta$ describing the dynamical coupling of the nuclear surface to the bulk. The inertial as well as the restoring parameters are both shown to be quadratic functions of this parameter $\beta$. The velocity fields and the inertia tensor are evaluated directly from an integration of the continuity equation. The variational densities and the restoring parameter matrix are calculated using the realistic Skyrme-type interaction $\mathrm{SkM}^{*}$ fitted to binding energies and densities of stable nuclei as well as to fission barriers of actinide nuclei.

The energies of the constrained modes as a function of the parameter $\beta$ exhibit two characteristic extrema, a minimum in the region of $\beta \sim-0.3$ (scaling type density vibration) for practically all nuclei, and a maximum at a value of $\beta$ which corresponds to an antiscaling behaviour of the density. Treating the motion as a coupled vibration of bulk and surface regions, the normal modes are identified, according to Rayleigh's principle, as the modes belonging to the two stationary energies obtained for the constrained motion.

The encrgies of the lower normal mode are in exccllent agreement with the empirical GMR energies, which obviously is a consequence of the fact that we used an appropriate parametrization of the nuclear densities and a realistic interaction. They are situated only little below the pure bulk density compression mode energies obtained for $\beta=0$.

The second normal mode lies higher in energy than the first one by an amount of about $11 \mathrm{MeV}$, remarkably constant over the whole range of nuclei. It also lies appreciably above the pure surface mode $(\beta= \pm \infty)$. For the estimation of the observability of this mode, calculations of strength functions and sum rules seem to be necessary, which is out of the scope of the present paper. In the picture of the forced excitation of two coupled oscillators, this second breathing mode might have some influence on the shape and width of the first resonance (and vice versa), irrespective of its dependence on decays into non-collective internal degrees of 


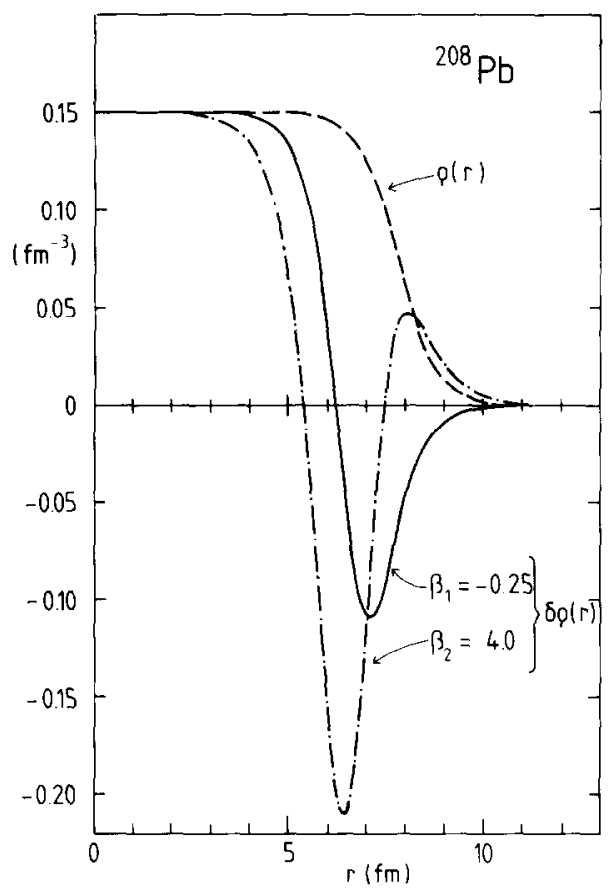

Fig. 7. Transition densities $\delta \rho(r)$ of ${ }^{208} \mathrm{~Pb}$ corresponding to the lower $\left(\beta_{1}=-0.25\right.$, solid line) and the upper $\left(\beta_{2}=4.0\right.$, dashed-dotted line) breathing eigenmode. Note the two nodes of the latter curve, which are typical for an antiscaling behaviour $(\beta>0)$. The total ground-state density is also shown for comparison (dashed line).

freedom or to the continuum. It is characterized by an antiscaling behaviour of its transition density which shows up with two nodes, as can be seen in fig. 7. This property might be used to identify it amongst many excitation modes [e.g. isovector monopole ${ }^{9.10}$ ) and higher multipolarity modes] lying in the energy region where it is predicted.

\section{Appendix A}

We shall use the standard "leptodermous expansion" ${ }^{27}$ ) to derive asymptotic expressions for the velocity field $v(r, q)$ defined by eqs. (27), (28). We start from the density $\rho(r, q)$, defined by eqs. $(8)-(10)$ :

$$
\rho(r, q)=\frac{\rho_{0} q}{1+\exp \left[(r-R) / \alpha_{0} q^{\beta}\right]} .
$$

The integral for the number of particles,

$$
\int \mathrm{d}^{3} r \rho(r, q)=A,
$$


may be expanded in powers of $(\alpha / R)$. Inverting the so-obtained expression, one gets the following expansion for the half-density radius $R$ in powers of $A^{-1 / 3}$ :

with

$$
R=r_{0}(q) A^{1 / 3}-\frac{\pi^{2} \alpha_{0}^{2} q^{2 \beta}}{3 r_{0}(q)} A^{-1 / 3}+\cdots
$$

$$
r_{0}(q)=\left(\frac{4}{3} \pi \rho_{0} q\right)^{-1 / 3} .
$$

Higher-order terms are not necessary in eq. (A.2) for practical applications with $A>40$.

Next we write down an expression for $\partial \rho / \partial q$ :

where

$$
\frac{\partial \rho}{\partial q}=\frac{1}{q} \rho(r, q)+\frac{\partial}{\partial r} \rho(r, q)\left[D-\frac{\beta r}{q}\right]
$$

$$
D=\frac{\beta}{q} R-\frac{\partial R}{\partial q}
$$

With eq. (A.2) we get an expansion for $D$ :

$$
D=\frac{1}{q}\left(\beta+\frac{1}{3}\right) \tilde{R}
$$

with

$$
\tilde{R}=r_{0} A^{1 / 3}+\frac{\pi^{2} \alpha_{0}^{2} q^{2 \beta}}{3 r_{0}} A^{-1 / 3}+\cdots .
$$

Note that $D$ eq. (A.6) is exactly equal to zero for the scaling mode, $\beta=-\frac{1}{3}$.

We want now to find approximate solutions for the velocity field $v$ from the continuity equation in the spherical case:

$$
\frac{\partial \rho}{\partial q}+v \frac{\partial \rho}{\partial r}+\rho \operatorname{div} v=0 .
$$

We insert eq. (A.4) into eq. (A.8), perform the spatial derivatives of $\rho$ (at $q=1$ ) and expand the whole equation (A.8) in powers of $\mathrm{e}^{-|x|}$ for large absolute values of $x$ :

$$
x=(r-R) / \alpha_{0} .
$$

Expanding at the same time $v(r, q=1)$ around $r=0$, we find, for all values of $\beta$,

$$
v(r, q=1)=-\frac{1}{3} r+\left(\beta+\frac{1}{3}\right) \mathrm{O}\left(\mathrm{e}^{x}\right), \quad \text { for } r \ll R .
$$

Similarly, the asymptotic behaviour of $v$ in the outer surface of the nucleus is found to be

$$
v(r, q=1)=v_{0}+\beta r+\left(\beta+\frac{1}{3}\right) \mathrm{O}\left(\mathrm{e}^{-x}\right), \quad \text { for } r \gg R,
$$


with

$$
v_{0}=\left(\beta+\frac{1}{3}\right)\left[-R+3 \alpha_{0}+\mathrm{O}\left(A^{-1 / 3}\right)\right]
$$

\section{Appendix B}

We shall prove here the equivalence of diagonalizing the two-dimensional hamiltonian (eq. (37)) with our use of the "frozen" parameter $\beta$ and its determination by the variational principle eq. (36). To simplify the notation, we define

$$
\begin{array}{lll}
B_{1} \equiv B_{\rho \rho}, & B_{2} \equiv B_{\alpha \alpha}, & B_{\Delta}=B_{\alpha \rho}, \\
K_{1} \equiv K_{\rho \rho}, & K_{2} \equiv K_{\alpha \alpha}, & K_{\Delta}=K_{\alpha \rho} .
\end{array}
$$

Solving the eigenmode equation (38) means diagonalization of the real matrix

$$
B^{-1} K=\Omega=\left(\begin{array}{ll}
a_{1} & a_{\Delta} \\
a_{\Delta} & a_{2}
\end{array}\right)
$$

where

$$
\begin{gathered}
a_{1}=\frac{1}{\Delta_{B}}\left(B_{2} K_{1}-B_{\Delta} K_{\Delta}\right), \\
a_{2}=\frac{1}{\Delta_{B}}\left(B_{1} K_{2}-B_{\Delta} K_{\Delta}\right), \\
a_{\Delta}=\frac{1}{\Delta_{B}}\left(B_{2} K_{\Delta}-K_{2} B_{\Delta}\right) \equiv \frac{1}{\Delta_{B}}\left(B_{1} K_{\Delta}-K_{1} B_{\Delta}\right), \\
\Delta_{B}=\operatorname{det} B=B_{1} B_{2}-B_{\Delta}^{2} .
\end{gathered}
$$

The identity eq. (B.4) follows from the fact that two real symmetric matrices, at least one of which is positive definite, always commute ${ }^{31}$ ).

The eigenvalues $\lambda_{i}=\omega_{i}^{2}$ of $\Omega$ eq. (B.2) are readily written down:

$$
\lambda_{1,2}=\frac{1}{2}\left(a_{1}+a_{2}\right) \pm \sqrt{\left(\frac{1}{2}\left(a_{1}+a_{2}\right)\right)^{2}-\operatorname{det} \Omega} .
$$

Denoting the eigenvectors by $\boldsymbol{x}_{i}$,

$$
\boldsymbol{x}_{i}=\left(\begin{array}{l}
x_{i 1} \\
x_{i 2}
\end{array}\right)
$$

we obtain for the ratio of their components

$$
\frac{x_{i 2}}{x_{i 1}}=\frac{\lambda_{i}-a_{1}}{a_{\Delta}}=\frac{a_{2}-a_{1}}{2 a_{\Delta}} \pm \sqrt{\left(\frac{a_{1}-a_{2}}{2 a_{\Delta}}\right)^{2}+1} .
$$

We now claim that the ratio $x_{i 2} / x_{i 1}$ of the amplitudes is identical with the values of $\beta$ at the stationary points of $\hbar \omega(\beta)$, eq. (35). To prove this we just perform the 
differentiation in

$$
\left.\frac{\partial \omega)^{2}}{\partial \beta}\right|_{\beta_{i}}=0
$$

explicitly, which leads after some manipulations, using the identity (B.4), to the quadratic equation

$$
\beta_{i}^{2}+2 \beta_{i}\left(a_{1}-a_{2}\right) / 2 a_{\Delta}-1=0
$$

with the solutions

$$
\beta_{1,2}=\bar{\beta} \pm \sqrt{\bar{\beta}^{2}+1}
$$

where

$$
\bar{\beta}=\left(a_{2}-a_{1}\right) / 2 a_{\Delta} .
$$

Thus, $\beta_{i}$ (eq. (B.11)) is indeed identical with $x_{i 2} / x_{i 1}$ (eq. B.8)). We see also from eqs. $(B .10,11)$ that

$$
\beta_{1} \beta_{2}=-1,
$$

which expresses the fact that the two eigenvectors are orthogonal. It is interesting also to note that $\bar{\beta}$ (eq. (B.12)), and therefore also the $\beta_{i}$, can be expressed in terms of the elements of either the compression modulus $K_{i j}$ or the inertial tensor $B_{i j}$ alone:

$$
\bar{\beta} \equiv \frac{B_{2}-B_{1}}{2 B_{\Delta}} \equiv \frac{K_{2}-K_{1}}{2 K_{\Delta}} .
$$

Finally, it is just a matter of some algebra to verify that inserting $\beta_{i}$ into $\hbar \omega(\beta)$ (eq. (35)), one obtains exactly the energies $\hbar \sqrt{\lambda_{i}}$ of the eigenmodes according to eq. (B.6) [see also ref. $\left.{ }^{28}\right)$ ]. One also can verify that the curve $\hbar \omega(\beta)$ cuts its horizontal asymptote at the point $\bar{\beta}$ (see also fig. 4):

$$
\hbar \omega(\bar{\beta})=\lim _{\beta \rightarrow \pm \infty} \hbar \omega(\beta)=\hbar \sqrt{K_{\alpha \alpha} / B_{\alpha \alpha}} .
$$

\section{References}

1) J. Speth and A. van der Woude, Reports Progr. Phys. 44 (1981) 719

2) K. Goeke and J. Speth, Ann. Rev. Nucl. Part. Sci. 32 (1982) 65

3) J.P. Blaizot, Phys. Reports 65 (1980) 171

4) B.K. Jennings and A.D. Jackson, Phys. Reports 66 (1980) 141

5) G.F. Bertsch and S.F. Tsiai, Phys. Reports 18 (1975) 125

6) Y. Abgrall and E. Caurier, Phys. Lett. 56B (1975) 229;

S. Krewald, R. Rosenfelder, J.E. Galonska and A. Faessler, Nucl. Phys. A269 (1976) 112

7) O. Bohigas, A.M. Lane and J. Martorell, Phys. Reports 51 (1979) 267;

K. Goeke, B. Castel and P.G. Reinhard, Nucl. Phys. A339 (1980) 339

8) G. Bertsch, Nucl. Phys. A249 (1975) 253;

F.E. Serr, G. Bertsch and J. Borysowicz, Phys. Lett. 92B (1980) 241 
9) G. Eckhart, G. Holzwarth and J.P. da Providencia, Nucl. Phys. A364 (1981) 1, and references quoted therein

10) A.S. Jensen and S.M. Larsen, Phys. Scripta 24 (1981) 534

11) J. Treiner, H. Krivine, O. Bohigas and J. Martorell, Nucl. Phys. A371 (1981) 253

12) C. Guet, H.B. Håkansson and M. Brack, Phys. Lett. 97B (1980) 7

13) M. Brack, C. Guet, H.-B. Hăkansson, A. Magner and V.M. Strutinsky, 4th Conf. on nuclei far from stability, Helsingør, 1981 (CERN 81-09, Geneva) p. 65

14) M. Brack, C. Guet and H.-B. Håkansson, to be published;

see also M. Brack, Méthodes mathématiques de la physique nucléaire, ed. M. Demeur, B. Giraud and $\mathrm{Ph}$. Quentin (Université Libre de Bruxelles, 1982) p. 251

15) J. Bartel, P. Quentin, M. Brack, C. Guet and H.-B. Håkansson, Nucl. Phys. A386 (1982) 79

16) H. Krivine, J. Treiner and O. Bohigas, Nucl. Phys. A366 (1980) 155

17) M. Brack and W. Stocker, Nucl. Phys. A388 (1982) 230

18) M. Farine, J. Côté, J.M. Pearson and W. Stocker, Z. Phys. A309 (1982) 151

19) B. Grammaticos and A. Voros, Ann. of Phys. 129 (1980) 153

20) C. Guet and M. Brack, Z. Phys. A297 (1980) 247

21) M. Brack and P. Quentin, Nucl. Phys. A361 (1981) 35

22) M. Beiner, H. Flocard, Nguyen Van Giai and P. Quentin, Nucl. Phys. A238 (1975) 29

23) Y. Abgrall, B. Morand, E. Caurier and B. Grammaticos, Nucl. Phys. A346 (1980) 431

24) A.S. Jensen and S.E. Koonin, Phys. Lett. 73B (1978) 243

25) J.W.S. Rayleigh, Theory of sound (Dover, New York, 1945) p. $109 \mathrm{ff}$.

26) G.F. Bertsch, Ann. of Phys. 86 (1974) 138

27) W.D. Myers and W.J. Swiatecki, Ann. of Phys. 55 (1969) 395

28) L.D. Landau and E.M. Lifshitz, Mechanics, a course of theoretical physics, vol. I (Pergamon, NY, 1959) sect. 23

29) P. Ring and P. Schuck, The nuclear many-body problem (Springer, New York, 1980) sect. 13.3

30) H.P. Morsch, C. Süskösd, M. Rogge, P. Turek, H. Machner and C. Mayer-Böricke, Phys. Rev. C22 (1980) 489;

H.P. Morsch and P. Decowski, private communications

31) R. Courant and D. Hilbert, Methods of mathematical physics, vol. I (Wiley, New York, 1953) sect. 5. 